la memoria activa. Se empleó una versión paramétrica de la prueba basada en tareas de manipulación de la información (N-Back Task) con el objetivo de determinar: a) si había diferencias entre la memoria activa de los no fumadores y la de los fumadores (en abstinencia o saciados); b) si la memoria activa de los fumadores se afectaba después de $\geq 13 \mathrm{~h}$ de abstinencia; y c) si fumar un cigarrillo mejoraba el funcionamiento de la memoria activa de los fumadores después de $\geq 13$ horas de abstinencia.

En el estudio participaron 15 fumadores y 22 no fumadores, pareados según sus datos demográficos. Los fumadores consumían más de 15 y menos de 40 cigarrillos diarios, eran mayores de 18 años y menores de 55, no utilizaban drogas llamadas recreativas (excepto marihuana, una vez a la semana como máximo) ni tomaban más de 10 bebidas alcohólicas por semana. Además, declararon no haber tomado medicamentos antidepresivos, estimulantes o somníferos ni haber sufrido traumas craneanos. Los no fumadores habían consumido menos de cinco cigarrillos en toda su vida.

Las pruebas con tareas de manipulación de la información se administraron en dos bloques diarios durante dos días. La prueba a los fumadores después de $\geq 13$ h sin fumar (sesión de abstinencia) y la prueba después de $\leq 1 \mathrm{~h}$ de haber fumado (sesión a saciedad) se realizaron en días diferentes. La mitad de los fumadores tuvieron la sesión de abstinencia el primer día y la otra mitad la tuvo el segundo. En ambas sesiones, los fumadores fumaron un cigarrillo entre los dos bloques de pruebas y antes de cada bloque se midió la ansiedad por fumar. Antes de la prueba a saciedad, los fumadores pudieron fumar ad libitum antes de llegar al laboratorio.

En la sesión a saciedad, el tiempo de respuesta de los fumadores no difirió significativamente del de los no fumadores $(F(1,32)=0,34 ; P=$ $0,57)$ como tampoco difirieron según la tasa de errores $(F(1,32)=0,17 ; P=0,68)$. Sin embargo, en la sesión de abstinencia, los fumadores tuvieron una tasa de errores significativamente mayor que los no fumadores $(F(1,32)=6,8 ; P=0,013)$. No se encontró diferencia entre ambos grupos en cuanto al tiempo de respuesta $(F(1,32)=1,7 ; P=2,0)$.

Los fumadores realizaron las tareas de manipulación de la información más lentamente $(F(1,14)=$ $5,18 ; P=0,039)$ y con una mayor tasa de errores $(F(1,14)=5,73 ; P=0,032)$ durante la sesión de abstinencia que en la sesión a saciedad. Además, los fumadores mostraron síntomas sicológicos y físicos más negativos después de $\geq 13$ h de abstinencia que durante la sesión a saciedad. No se observó que los antecedentes del hábito de fumar influyeran en los resultados de los fumadores.
Este estudio demostró que el abandono súbito del hábito de fumar afectó a la memoria activa en un grupo de fumadores con dependencia moderada o intensa a la nicotina. Se observó que un período de abstinencia $\geq 13 \mathrm{~h}$ provocó un déficit significativo en la precisión de las tareas realizadas en comparación con los no fumadores y más lentitud de la habitual en realizarlas. Estos resultados indican que la pérdida de algunas habilidades es consecuencia del cese abrupto del hábito de fumar y respaldan la hipótesis de que esta afectación puede influir negativamente en la intención de muchos fumadores de dejar el hábito. (Mendrek A, et al. Working memory in cigarette smokers: comparison to non-smokers and effects of abstinence. Addict Behav. 2006;31(5):833-44.)

\section{Efectos de la infección por Helicobacter pylori en el peso y la talla de los niños colombianos}

La infección por Helicobacter pylori provoca diversas afecciones crónicas del sistema digestivo y está asociada con la diarrea y la desnutrición crónicas. Esta infección se adquiere por lo general durante la infancia, y en los países en desarrollo su prevalencia es elevada en niños pequeños, especialmente en los que sufren desnutrición y retraso del crecimiento.

En este artículo se discute si una nueva infección con $H$. pylori tiene efectos temporales o permanentes en el crecimiento de los niños en edad preescolar. Para ello se realizó un estudio de cohorte con 521 niños de 12 a 60 meses de edad seleccionados entre los que asistían a tres guarderías infantiles de la ciudad de Cali, Colombia, entre 1997 y 2001. Los niños gozaban de buena salud en general y no padecían ninguna enfermedad crónica. Se realizó un seguimiento (mediana: 494 días; recorrido de 257 a 811 días) de los 347 niños con resultados negativos en la prueba de respiración con ${ }^{13} \mathrm{C}$ para detectar la infección por H. pylori. Se tomaron los datos demográficos, socioeconómicos y sobre la higiene doméstica en el momento de entrar al estudio. Las mediciones antropométricas se hicieron en el momento inicial y cada 2 meses.

En general, 105 niños (30,3\%) tuvieron resultados positivos en la prueba detectora de $H$. pylori al menos durante un período de 2 meses, mientras que 242 niños $(69,7 \%)$ se mantuvieron sin infección durante todo el tiempo de seguimiento.

Se observó una reducción estadísticamente significativa del crecimiento durante los primeros 4 meses de la infección. El efecto adverso de una nueva infección se manifestó de forma aguda y sin demora y se mantuvo inalterado durante los si- 
guientes 4 meses, atenuándose paulatinamente hasta casi desaparecer en el octavo mes. A largo plazo, el efecto de la nueva infección dependió de la edad a la que se detectó la infección por primera vez y se observó un efecto acumulativo a medida que pasaba el tiempo. La talla perdida no se recuperó y al llegar al octavo mes, la diferencia acumulada era de 0,14 cm (intervalo de confianza de $95 \%$ [IC95\%]: 0,11 a 0,17 cm) en relación con los niños sin la infección. Debido a que los niños menores crecen más rápidamente, cuando la infección ocurrió antes de los 12 meses de edad, los niños perdieron en promedio $0,25 \mathrm{~cm}$ de talla en los primeros 8 meses de infección (IC95\%: 0,21 a 0,29 cm).

Con relación al peso, se encontró una pérdida de peso pequeña pero significativa inmediatamente después de establecerse la infección, aunque desapareció a la siguiente visita de control. Sin embargo, no se observó un incremento compensatorio en los siguientes meses. De esta forma, se comprobó que el efecto de la infección con $H$. pilori sobre el peso del niño es menor y de menos duración que sobre su estatura. Esto puede deberse a la naturaleza crónica de esta infección y a que el peso presenta por lo general una mayor variabilidad.

Los resultados de este trabajo indican que el efecto de la infección con $H$. pylori sobre la talla de los niños de edad preescolar es mayor inmediatamente después de la infección y decrece aproximadamente a la mitad a los 8 meses de seguimiento. El retraso del crecimiento fue acumulativo y no se recuperó, por lo que la diferencia se mantuvo al menos durante todo el período de seguimiento. El retraso acumulado del crecimiento puede deberse a las altas tasas de reinfección en algunos niños. La aplicación de medidas dirigidas a prevenir y eliminar esta infección en niños de edad preescolar puede evitar retrasos del crecimiento. (Mera RM, et al. Effects of a new Helicobacter pylori infection on height and weight in Colombian children. Ann Epidemiol. 2006;16:347-51.)

\section{Factores asociados con el estado nutricional en niños de 0 a 23 meses en Nicaragua}

Nicaragua es un país pobre con una alta prevalencia de enfermedades infecciosas y desnutrición. En este artículo se examina el estado nutricional de los niños de 0 a 23 meses en varias zonas urbanas y rurales de Nicaragua y se identifican los factores asociados con la desnutrición infantil en el país.

Se realizó un estudio transversal en el municipio de Nadine, provincia de Granada, Nicaragua, entre febrero y marzo de 2002. Se tomaron las medidas antropométricas (peso y talla) de 756 niños y se aplicó un cuestionario estructurado a sus madres. En el cuestionario se preguntó acerca de los episodios de fiebre y las enfermedades diarreicas y respiratorias que habían padecido los niños en los 14 días previos a la encuesta. Como indicadores del estado nutricional se calcularon las puntuaciones $Z$ ( $Z$ scores) de la relación peso-edad (ZPE), de la relación talla-edad (ZTE) y de la relación peso-talla (ZPT).

La proporción de niños con bajo peso (ZPE $<-2$ desviaciones estándar [DE]) fue de 10,3\%, mientras que $30,1 \%$ de los niños tenían retraso del crecimiento (ZTE $<-2 \mathrm{DE})$ y $5,0 \%$ presentaban atrofia $(\mathrm{ZPT}<-2 \mathrm{DE})$.

Se encontraron diferencias significativas $(P<$ $0,05)$ entre la ZPE de los niños de zonas urbanas $(0,37 ; \mathrm{DE}=1,26)$ y rurales $(-0,54 ; \mathrm{DE}=1,27)$. La ZTE promedio fue de $-1,01$ ( $\mathrm{DE}=1,66)$ en las zonas urbanas y de $-1,36(\mathrm{DE}=1,56)$ en las rurales $(P<0,05)$. Los niños de zonas rurales estaban más propensos a tener retraso en el crecimiento $(P<0,05)$. No se encontraron diferencias significativas entre las ZPT de los niños de las zonas urbanas y rurales (5,8 y 4,1, respectivamente).

Los factores de riesgo de retraso en el crecimiento fueron el tener una madre analfabeta o que no había recibido educación institucional (razón de posibilidades [odds ratio, RR] $=3,476 ; P<0,01$ ), haber sufrido alguna infección respiratoria $(\mathrm{RR}=$ 1,821; $P<0,05)$ o haber tenido bajo peso ( $<3000 \mathrm{~g})$ al nacer $(\mathrm{RR}=1,704 ; P<0,05)$. Los factores de riesgo del bajo peso fueron haber tenido alimentación materna por más de 12 meses $(R R=2,031 ; P<0,01)$, no haber tenido seguimiento del crecimiento $(\mathrm{RR}=$ $1,956 ; P<0,05)$ y ser de sexo femenino $(\mathrm{RR}=1,884$; $P<0,05)$.

Según los resultados del análisis bifactorial, los factores asociados con el retraso del crecimiento (ZTE $<-2$ DE) fueron: bajo ingreso mensual $(P<$ $0,01)$, tener una madre analfabeta o sin educación institucionalizada $(P<0,01)$, haber recibido alimentación materna por más de 12 meses $(P<0,01)$, vivir en la ciudad $(P<0,05)$, ser de sexo femenino $(P<$ $0,05)$, haber nacido con bajo peso $(P<0,001)$ y haber sufrido alguna infección respiratoria $(P<0,05)$. El retraso del crecimiento estuvo asociado con las condiciones socioeconómicas de la familia más que con la conducta materna de garantizar la salud, como mantener el seguimiento del crecimiento de su hijo o participar en actividades de educación sanitaria, entre otras.

En cuanto al bajo peso (ZPE <-2 DE), según el análisis bifactorial, los factores de riesgo fueron: la lactancia materna por más de 12 meses $(P<0,01)$, ser de sexo femenino $(P<0,01)$, haber nacido con bajo peso $(P<0,05)$ y no haber tenido seguimiento del crecimiento $(P<0,01)$. 